\title{
Human skeletal muscle fibre type grouping and collateral re-innervation
}

\author{
C. J. MORRIS \\ From the Department of Pathology, Midland Centre for Neurosurgery and Neurology, Smethwick
}

Human skeletal muscle contains at least two fibre types (Engel, 1962) recognizable by histochemical techniques. Type 1 fibres have a strong reaction for reduced diphosphopyridine-nucleotide diaphorase and lactate dehydrogenase, and a weak one for phosphorylase and myosin adenosine triphosphatase, whereas type 2 fibres show a reciprocal reaction to that of type 1 .

In transverse sections of normal human skeletal muscle, types 1 and 2 fibres are distributed in a random fashion (Fig. 1), the number of fibres of the same type in contact with one another rarely exceeding 15 (Engel, 1965). In some chronic peripheral neuropathies, this pattern is changed by a marked grouping of one or other of the two fibre types, groups of up to 100 fibres of the same histochemical type being encountered (Fig. 2). It has been suggested that this results from re-innervation of denervated muscle fibres by sprouting axons (Engel, 1965; Karpati and Engel, 1968a), but no direct evidence of this in human muscle has so far been reported in the literature.

\section{MATERIALS AND METHODS}

Limb muscle from two normal cases and 12 patients with neuromuscular disease was obtained by motor point biopsy (Coërs and Woolf, 1959a). A strip of muscle $(1 \mathrm{~cm} \times 0.5 \mathrm{~cm})$ was taken for the histochemical procedures after rapid freezing in liquid nitrogen. A second strip of larger dimensions $(0.75 \mathrm{~cm} \times 3 \mathrm{~cm}$ approx.) was removed after vital staining with methylene blue (Coërs, 1952; Woolf, 1962). Transverse sections of the first specimen, $6 \mu$ thick, were cut on a cryostat with a cabinet temperature of $-20^{\circ} \mathrm{C}$, attached directly to cooled microscope slides and incubated for reduced diphosphopyridine nucleotide diaphorase (Scarpelli, Hess, and Pearse, 1958). This method was chosen as it gave excellent differentiation between fibre types 1 and 2 with minimum handling of the tissue. The demonstration of myofibrillar adenosinetriphosphatase is probably the most reliable way of differentiating the two fibre types (Karpati and Engel, $1968 \mathrm{~b}$ ), but necessitates much handling of the sections, an undesirable practice when the quantitative procedures to be used require as complete a section as possible. The sections were mounted in Polyvinyl-pyrrolidone (Bur- stone, 1957), examined under the light microscope and selected for study if they had no histochemical abnormality other than grouping.

The muscle from 14 patients satisfied these criteria, cases 1 to 9 having a completely normal appearance and cases 10 to 14 showing grouping. In each of the latter five cases, there was clinical evidence of degeneration of the lower motor neurone. The sections were photographed and printed to give a final linear magnification of $\times 112$ on normal bromide paper size $12 \times 10$ in. The distribution of the fibre types 1 and 2 in the sections was then assessed by a square-counting method, the magnification having been chosen so as to ensure that most of the orige inal section was included in the counting process. Onl one section representing the whole diameter of the muscles strip was used for each clinical case, as the length of the muscle cell is such that sections cut further along the length of such a small sample as the biopsy specimero would have essentially the same appearance. Each photoe graphic print was laid flat and covered by a thin sheet of clear film base, size $10 \times 8$ in., with a grid of 1 in squares drawn on it. The number of type 1 and 2 fibres in each square was then counted. It was found by experience that 48 such squares, each containing approximately 15 muscle fibres, could be counted, allowing for spaces in the section containing tissue elements other than muscle and for fluid spaces between fasciculi, which made the squares they traversed unsuitable for counting. The number of type 1 fibres was then expressed as a percentage of the total number of fibres for each square, and the results were put into histogram form (Figs. 3 to 6 ) around a calculated mean percentage of type 1 fibres for the whole section.

The vitally stained preparations were examined under the light microscope and the number of muscle fibres innervated by a known number of axons was calculated for each clinical case, as many axons as possible being assessed in each case. The functional terminal innervation ratio (FTIR) was then calculated from these figures according to the method of Coërs and Woolf (1959b).

\section{RESULTS}

ENZYME HISTOCHEMISTRY OF TISSUE SECTIONS MUScles without fibre type grouping Clinical cases 1 to 9 all fall into this category, none of them having groups of muscle fibres of one histochemical type 


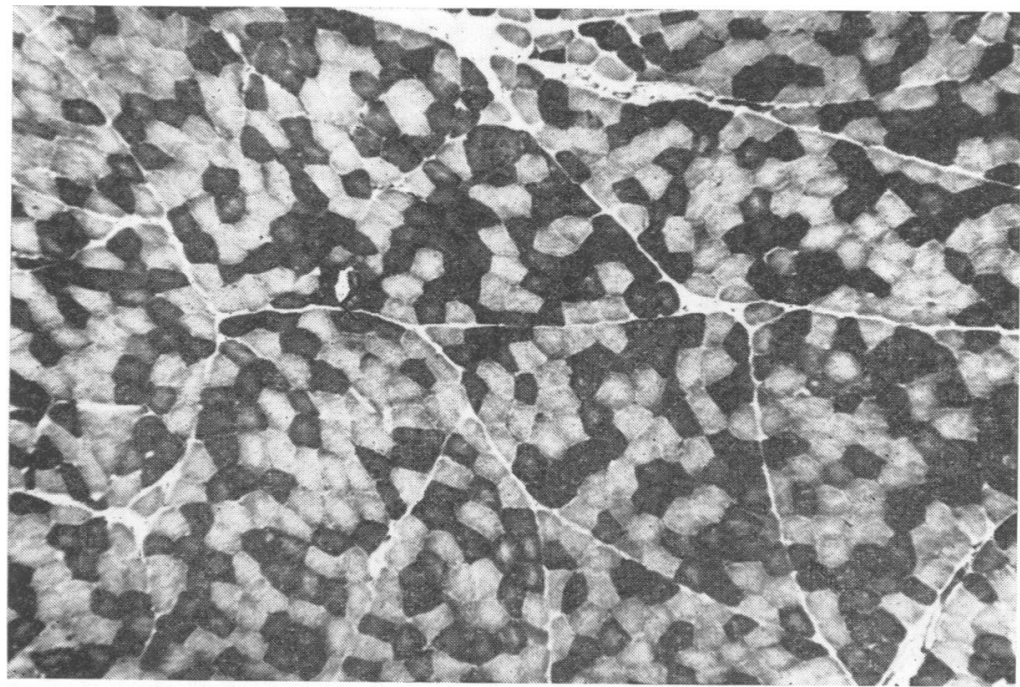

FIG. 1. Transverse section, human deltoid muscle (case 1, clinically normal), showing random distribution of type 1 (dark) and type 2 (light) fibres. Reduced diphosphopyridine nucleotide diaphorase DPNH-D, $\times 40$.

exceeding about 15 fibres. The arithmetical mean percentages of type 1 fibres range from 37.9 to $55.9 \%$ (Table), but in all but two cases the values lie between 49 and $55 \%$.

The histograms drawn from the square counts on these cases show a normal type of distribution (Fig. 3). Some of the histograms, however, have a slight positive skewness (Fig. 4).

Muscles showing marked grouping Clinical cases 10 to 14 all have large collections of type 1 fibres. The arithmetical means in these cases vary between 64 and $93 \%$, all but one lying in the range 64 to $74 \%$. These means are considerably higher than those in the non-grouped muscles, the difference between the lowest grouped mean and the highest non-grouped being $10 \%$. The histograms drawn from the results of the square counts on these cases (Fig. 5) show that the distribution of the type 1 fibre percentages about the mean is not normal, but shows a wide range of values with several peaks.

VITALLY STAINED PREPARATIONS The results of the axon and innervated muscle fibre counts from the methylene blue preparations are summarized in the Table. There is a marked difference between the grouped cases 10 to 14 and cases 1 to 9 , these nongrouped cases having FTIR values between $1 \cdot 11$

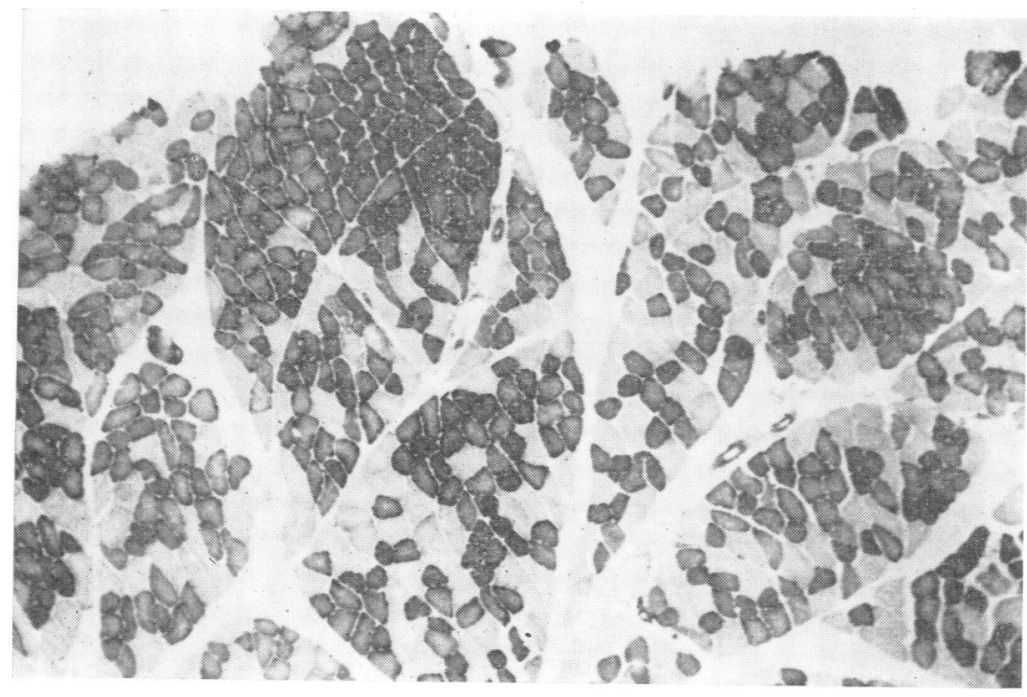

FIG. 2. Transverse section, human flexor carpi radialis muscle (case 10-peroneal muscular atrophy) showing marked grouping of type 1 fibres. DPNH-D, $\times 40$. 


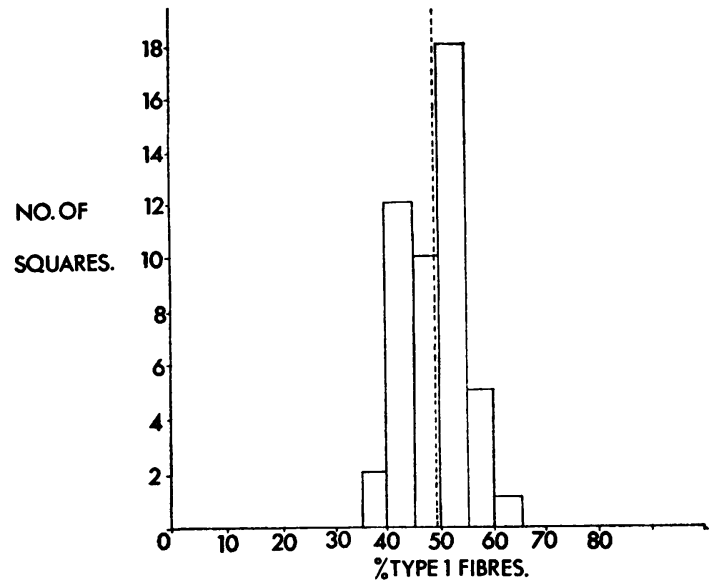

FIG. 3. Histogram of non-grouped muscle (case 6carcinomatous neuropathy). Broken vertical line-arithmetical mean percentage type 1 fibres.

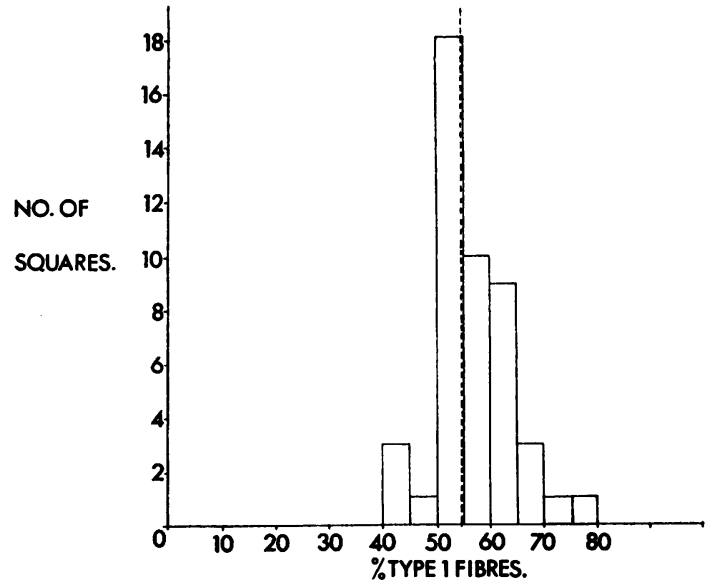

FIG. 4. Histogram of non-grouped muscle showing positive skewness (case 7-motor neurone disease).

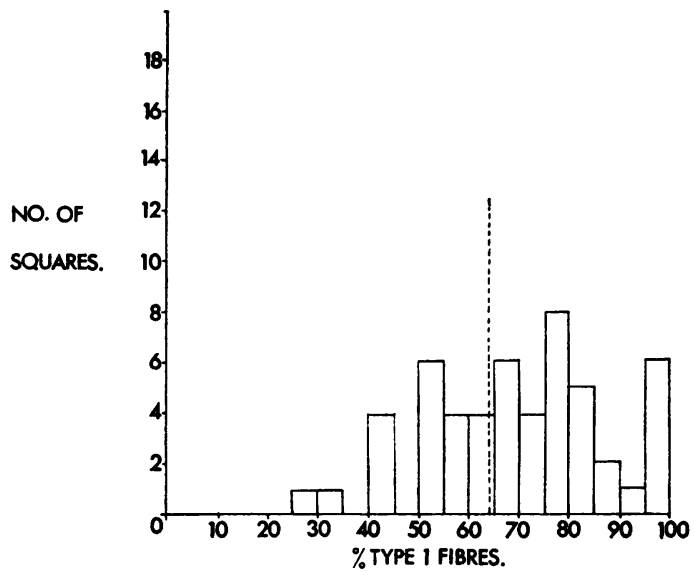

FIG. 5. Histogram of grouped muscle (case 11-polyarteritis nodosa).

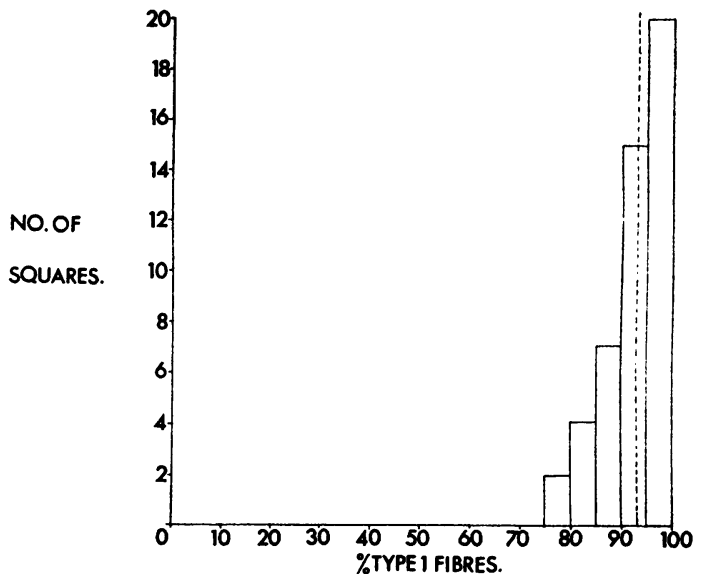

FIG. 6. Histogram of muscle with very extensive type 1 fibre grouping (case 14-carcinomatous neuropathy).

TABLE I

SUMMARY OF RESULTS FROM VITAL STAINING AND HISTOCHEMISTRY

\begin{tabular}{|c|c|c|c|c|c|}
\hline $\begin{array}{c}\text { Case } \\
\text { no. }\end{array}$ & Diagnosis & Muscle & $\begin{array}{c}\text { Subterminal } \\
\text { axons } \\
\text { (no.) }\end{array}$ & FTIR & $\begin{array}{c}\text { Arithmetical } \\
\text { mean }(\%) \\
\text { Type } 1 \text { fibres }\end{array}$ \\
\hline 1 & Normal & Deltoid & - & - & $49 \cdot 2$ \\
\hline 2 & Normal & Flexor carpi radialis & 72 & $1 \cdot 11$ & $45 \cdot \overline{9}$ \\
\hline 3 & Myositis & Flexor carpi radialis & 85 & $1 \cdot 10$ & $37 \cdot 9$ \\
\hline 4 & Guillain-Barré syndrome & Flexor digitorum sublimis & 83 & $1 \cdot 18$ & 55.9 \\
\hline 5 & Lumbar neurofibroma & Palmaris longus & 67 & $1 \cdot 24$ & $50 \cdot 9$ \\
\hline 6 & Carcinomatous neuropathy & Palmaris longus & 72 & $1 \cdot 12$ & $49 \cdot 3$ \\
\hline 7 & Peripheral neuropathy & Palmaris longus & 80 & $1 \cdot 26$ & $54 \cdot 8$ \\
\hline 8 & Amyotrophic lateral sclerosis & Palmaris longus & 65 & $1 \cdot 35$ & $55 \cdot 2$ \\
\hline 9 & Amyotrophic lateral sclerosis & Flexor carpi radialis & 71 & $1 \cdot 24$ & $49 \cdot 2$ \\
\hline 10 & Amyotrophic lateral sclerosis & Flexor carpi radialis & 31 & $1 \cdot 74$ & $64 \cdot 7$ \\
\hline 11 & Familial sensory neuropathy & Deltoid & 18 & $2 \cdot 17$ & $64 \cdot 0$ \\
\hline 12 & Post-gastrectomy neuropathy & Flexor carpi radialis & 58 & $2 \cdot 26$ & $\mathbf{7 4 \cdot 4}$ \\
\hline 13 & Alcoholic neuropathy & Tibialis anterior & 31 & $2 \cdot 20$ & $71 \cdot 4$ \\
\hline 14 & Carcinomatous neuropathy & Deltoid & 41 & $2 \cdot 51$ & $93 \cdot 5$ \\
\hline
\end{tabular}


and 1.35 compared with the 1.74 to 2.51 of the grouped cases.

\section{DISCUSSION}

The specimens examined in cases 1 to 9 were taken from four different muscles: deltoid, flexor carpi radialis, flexor digitorum sublimis, and palmaris longus. In all these muscles, with one exception, the sections obtained show that the mean percentage of type 1 fibres approximates to $50 \%$ of the total fibre count and that there is no significant difference between the means of the different muscles. The exception is case 3 in which there is a low mean percentage of type 1 fibres (37.9\%)-a difference that is probably not due to anatomical position as other biopsy specimens from the same muscle (case 2 and 9) have a mean percentage approximating to $50 \%$ type 1 fibres. It seems far more likely that the pathological process in the muscle (myositis) is the cause of the finding, as two more recent cases of this condition show the same low percentage of type 1 fibres. In animals, the ratios of the two fibre types do differ according to their position in the muscle, there being, for example, a difference between the medial and lateral heads of the gastrocnemius in the guinea-pig (Karpati and Engel, 1968b) and in the rat (Stein and Padykula, 1962), though there is no equivalent information for human muscle. This possible bias does not affect the results of this study, since all the material apart from the normal deltoid of case 1 was obtained from the motor point. The slight positive skewness in some of the non-grouped cases could be dependent on the relatively small number of fibres counted in each case. However, since it is not seen in all cases, it is more likely that it indicates a genuine tendency towards grouping of type 1 fibres. In the grouped cases, most of the groups consisted of type 1 fibres, and there did not seem to be any type 2 groups of comparable size. This is reflected statistically by the increase in the mean percentage of type 1 fibres, which also indicates that there is a net transformation of type 2 fibres to type 1 , and not a simple redistribution of the enzymatic characteristics of the original fibres with maintenance of their $50 \%$ proportions.

Of the histograms obtained from the grouped cases, only one (Fig. 6, case 14) shows any resemblance to a normal distribution curve; all the others were bi- or trimodal with peaks in the $50 \%$ and 85 to $100 \%$ range. Probably, if it had been possible to examine larger samples in all grouped cases, the distribution would have been bimodal, the peaks in the $50 \%$ range representing areas in which the original fibre distribution is maintained, and those in 85 to $100 \%$ range the grouped fibres. The one exception (case 14) shows a partial distribution curve which is attenuated at $100 \%$ because values do not go beyond this figure. In this type of histogram, the only distribution curve represented is that of grouped fibres, the grouping being so extensive that no areas with the original $50 \%$ distribution remain to produce the first part of the bimodal curve.

Collateral sprouting occurs in up to $10 \%$ of axons in normal human muscle, consequently visual assessment of small tissue samples unsupported by statistical analysis is insufficient to establish undoubted pathological re-innervation. The most satisfactory evidence is obtained by estimating the functional terminal innervation ratio (FTIR) in vitally stained preparations. Normal human limb muscles have an average FTIR of $1.09 \pm$ 0.089: 1 (Coërs, 1955a, b), values greater than 1.27: 1 are therefore outside the $95 \%$ range of the normally distributed population, and must be considered statistically abnormal. Of the 14 cases included in this study, the FTIR of cases 2, 3, 4, 5, 6,7 , and 9 are all within the normal statistical range, case 8 being marginally outside this figure. No methylene blue preparation was available for case 1 as the material was not obtained from the motor point. It may be that the innervation pattern in case 8 is genuinely abnormal, the increase in collateral sprouting being insufficient to cause any change in the histochemical appearance of the muscle.

Thus, our results show that muscles with a normal distribution of types 1 and 2 fibres have a normal innervation pattern with an FTIR approaching unity. In contrast to this, the grouped cases 10 to 14 all have FTIR values well outside the normally expected range, four of the cases having values more than double the normal mean. Very marked collateral re-innervation is therefore associated with fibre type grouping, the more marked grouping, -indicated by higher mean percentages of type 1 fibres-being associated with the higher FTIR values-that is, with more extensive re-innervation. As Karpati and Engel (1968a) have pointed out, there is no definite information concerning the connection between nerve supply and the histochemical appearances of the muscle and, in particular, as to whether all the muscle fibres innervated by a single anterior horn cell are invariably of the same histochemical type. This study does not remedy this deficiency, but it seems unlikely that grouping would be so closely correlated with collateral re-innervation if the muscle fibres reinnervated by collateral sprouts from a single axon usually maintained their original histochemical constitution. If transformation of muscle fibres 
from one histochemical type to another under the influence of re-innervating collateral sprouts is a possible explanation of grouping, the preponderance of type 1 groups might be due to a greater capacity for collateral re-innervation on the part of axons innervating type 1 fibres. Transformation of the histochemical profile of muscle fibres occurs in animals as a result of cross innervation (Dubowitz and Newman, 1967; Karpati and Engel, 1967; Romanul and Van Der Meulen, 1967). It should be mentioned here that personal studies not yet reported have indicated that not all collaterally re-innervated muscles show grouping. If this is confirmed, it would suggest that, in some cases, collateral re-innervation by itself results only in a change in the number and distribution of the muscle fibres innervated by a given (surviving) axon. Degeneration affecting only one fibre typeparticularly type 1-may explain the examples of fibre type grouping occasionally encountered in the absence of evidence of collateral re-innervation, as in our case 3. This was a case of myositis, and, as mentioned above, we have seen a similar type 2 grouping in two recent cases of this disease. However, we have not yet determined the FTIR in these cases, nor was it given for the case of chronic peripheral neuropathy in which type 2 fibre grouping is illustrated in a photograph by Karpati and Engel (1968a, Fig. 1). It should be mentioned that in none of the myositis cases were degenerated fibres observed.

\section{SUMMARY}

A combination of histochemistry and vital staining was carried out on limb muscle from 14 patients, 12 of whom had neuromuscular disease. The muscle from nine patients was histochemically normal and had a normal innervation pattern, as shown by a normal functional terminal innervation ratio. In the remaining five patients, the muscle showed grouping of type 1 fibres associated with collateral re-innervation and a much increased terminal innervation ratio.

It is tentatively suggested that type 1 grouping results from transformation of the histochemical properties of the muscle fibres under the influence of the terminal branches of axons participating in collateral re-innervation, the grouped distribution of the fibres being dependent on the changed terminal motor innervation pattern. In contrast, type 2 grouping may be a reflection of selective loss by degeneration of type 1 fibres.

My thanks are due to Dr. A. L. Woolf for performing the biopsies and for his help and advice. This work was supported by a grant from the Muscular Dystrophy Group of Great Britain.

\section{REFERENCES}

Burstone, M. S. (1957). Polyvinyl pyrrolidone as a mounting medium for stains for fat and for azo-dye procedures. Amer. J. clin. Path., 28, 429-430.

Coërs, C. (1952). The vital staining of muscle biopsies with methylene blue. J. Neurol. Neurosurg. Psychiat., 15, 211-215.

(1955a). L'exploration fonctionelle et l'étude histologique quantitative des muscles atrophiés. Acta clin. belg., 10, 244-265. (1955b). Les variations structurelles normales et pathologiques de la jonction neuromusculaire. Acta neurol. belg., 55, 741-866 and Woolf, A. L. (1959a). The Innervation of Muscle, pp. 1-7. Blackwell: Oxford.

- , - (1959b). The Innervation of Muscle, pp. 12-14. Blackwell Oxford.

Dubowitz, V., and Newman, D. L. (1967). Changes in enzyme patterm after cross innervation of fast and slow skeletal muscle Nature (Lond.), 214, 840-841.

Engel, W. K. (1962). The essentiality of histo- and cytochemical studies of skeletal muscle in the investigation of neuromusculas disease. Neurology (Minneap.), 12, 778-794.

(1965). Histochemistry of neuromuscular disease, significanc of muscle fibre types, pp. 67-74 in The Proceedings of the VIIIth International Congress of Neurology, Vienna.

Karpati, G., and Engel, W. K. (1967). Transformation of the histochemical profile of skeletal muscle by 'foreign' innervation. Nature (Lond.), 215, 1509-1510.

mental reinnervation. Neurology (Minneap.), 18, 447-455.

-1 - (1968b). Histochemical investigation of fibre type ratios with the myofibrillar ATP-ase reaction in normal and denervated skeletal muscles of guinea pig. Amer. J. Anat., 122, 145-155.

Romanul, F. C. A., and Van Der Meulen, J. P. (1967). Slow and fas muscles after cross innervation-enzymatic and physiologica changes. Arch. Neurol. (Chic.), 17, 387-402.

Scarpelli, D. G., Hess, R., and Pearse, A. G. E. (1958). The cytochemical localisation of oxidative enzymes. 1. Diphosphopyridine nucleotide diaphorase and triphosphopyridine nucleotide diaphorase. J. biophys. biochem. Cytol., 4, 747-751.

Woolf, A. L. (1962). Muscle biopsy, pp. 11-35 in Modern Trends in Neurology, series 3. Edited by D. Williams. Butterworth: London.

Stein, J. M., and Padykula, Helen A. (1962). Histochemical classification of individual skeletal muscle fibres of the rat. Amer. J. Anat., 110, 103-123. 